\title{
High-Pressure Phase Transformations under Severe Plastic Deformation by Torsion in Rotational Anvils
}

\author{
Valery I. Levitas ${ }^{1,2, *}$ \\ ${ }^{1}$ Departments of Aerospace Engineering and Mechanical Engineering, Iowa State University, Ames, Iowa 50011, USA \\ ${ }^{2}$ Division of Materials Science \& Engineering, Ames Laboratory, Ames, Iowa 50011, USA
}

\begin{abstract}
Numerous experiments have documented that combination of severe plastic deformation and high mean pressure during high-pressure torsion in rotational metallic, ceramic, or diamond anvils produces various important mechanochemical effects. We will focus here on four of these: plastic deformation (a) significantly reduces pressure for initiation and completion of phase transformations (PTs), (b) leads to discovery of hidden metastable phases and compounds, (c) reduces PT pressure hysteresis, and (d) substitutes a reversible PT with irreversible PT. The goal of this review is to summarize our current understanding of the underlying phenomena based on multiscale atomistic and continuum theories and computational modeling. Recent atomistic simulations provide conditions for initiation of PTs in a defect-free lattice as a function of the general stress tensor. These conditions (a) allow one to determine stress states that significantly decrease the transformation pressure and (b) determine whether the given phase can, in principle, be preserved at ambient pressure. Nanoscale mechanisms of phase nucleation at plastic-strain-induced defects are studied analytically and by utilizing advanced phase field theory and simulations. It is demonstrated that the concentration of all components of the stress tensor near the tip of the dislocation pileup may decrease nucleation pressure by a factor of ten or more. These results are incorporated into the microscale analytical kinetic equation for strain-induced PTs. The kinetic equation is part of a macroscale geometrically-nonlinear model for combined plastic flow and PT. This model is used for finite-element simulations of plastic deformations and PT in a sample under torsion in a rotational anvil device. Numerous experimentally-observed phenomena are reproduced, and new effects are predicted and then confirmed experimentally. Combination of the results on all four scales suggests novel synthetic routes for new or known high-pressure phases (HPPs), experimental characterization of strain-induced PTs under high-pressure during torsion under elevated pressure. [doi:10.2320/matertrans.MF201923]
\end{abstract}

(Received February 13, 2019; Accepted April 11, 2019; Published June 7, 2019)

Keywords: high-pressure torsion, strain-induced phase transformations, multiscale theory and simulations, rotational Bridgman anvils, rotational diamond anvils

\section{Introduction}

Large plastic deformations and their effects on various structural changes in solids have been intensively studied, starting with pioneering work by Bridgman. ${ }^{1-3)}$ A special device, rotating Bridgman's anvils made of hard alloys or metals was used for the study of plastic flow and grain refinement during plastic flow, ${ }^{2,4-6)}$ as well as to examine the effect of plastic shear on PTs ${ }^{1,3,7-14)}$ and chemical reactions. ${ }^{1,3,8,15,16)}$ At the same time, the high-pressure community studied material behavior, including PTs and chemical reactions in diamond anvils. As a merger of these two devices, rotational diamond anvils (Fig. 1) have been invented ${ }^{7,17)}$ and further developed. ${ }^{18)}$ An advantage of rotational diamond anvils is that they allow in situ study of pressure distribution ${ }^{7,19-22)}$ and distribution of the volume fraction of phases; ${ }^{21)}$ performance of optical, Ramam, and other investigations; and the achievement of much higher pressure, up to one hundred GPa.

Among multiple phenomena, we will focus on the following ones:

(1) Superposition of plastic straining on high pressure may result in the appearance of new (hidden) phases or reaction products that were not or could not be achieved under hydrostatic conditions. ${ }^{1,7,8,19,20,23-25)}$ In particular, superhard phase IV of fullerene was first obtained under shear in Refs. 7, 20) and confirmed in Ref. 19). We recently obtained a new high-density amorphous phase of $\mathrm{SiC}$, while amorphization is not

*Corresponding author, E-mail: vlevitas@iastate.edu

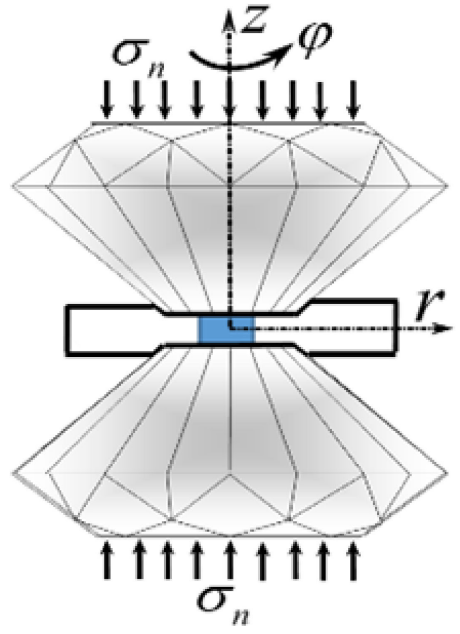

Fig. 1 Schematics of rotational diamond anvil cell. The sample is within a gasket compressed by diamond anvils. One of the anvils is then twisted under a constant load, superimposing large plastic deformation on high pressure. In some cases, the sample and gasket are of the same material.

observed even up to $135 \mathrm{GPa}$ under hydrostatic conditions.

(2) Plastic deformation decreases the pressure for initiation and completion of some PTs and chemical reactions, even up to an order of magnitude PTs. 7,8,15,16,19,20,23-27) For example, shear strain reduced the transformation pressure from 9 to $3 \mathrm{GPa}$ for $\mathrm{PT}$ from diamond-cubic Si I and Ge I to bcc Si III and Ge III; from 12.3 to $5.7 \mathrm{GPa}$ for PT from Si III to metallic Si II, and from 9.3 to 4.2 GPa Ge III $\rightarrow$ Ge II PT. ${ }^{7)}$ The largest transformation 
pressure drop, from 52.5 to $6.7 \mathrm{GPa}$, was obtained in Ref. 26) from hexagonal nanocrystalline $\mathrm{BN}$ with high concentration of the turbostratic stacking fault to wurtzitic BN, which is a superhard phase. Note that the thermodynamic equilibrium pressure for this PT is negative. $^{28)}$

(3) Plastic deformation may substitute a reversible PT with irreversible $P T^{1,3,7,8,21,27,29)}$ This allows one to utilize these metastable HPPs in various applications at ambient pressure.

(4) Plastic straining may reduce the pressure hysteresis (i.e. the difference between pressure for direct and reverse PTs), even to zero, e.g. for PTs in $\mathrm{KCl}^{7)} \mathrm{Zr}$, and $\mathrm{Ti}^{30)}$

(5) If plastic straining stops at relatively low pressure, the PT does not occur. ${ }^{7,8,19,20,23-27)}$ Thus, time is not a kinetic parameter and some measure of plastic strain governs the PT kinetics.

In this review paper, we analyze our current understanding of the above phenomena based on our developed multiscale theories and computer modeling. Note that we will not discuss the effect of plastic strain on diffusion and diffusive PTs here. Some reviews on this topic can be found in Refs. 7, $8,25)$.

\section{Classification of High-Pressure Transformations: Pressure- and Stress-Induced vs. Strain-Induced}

As was demonstrated in Ref. 31), traditional thermodynamics, even after substituting pressure and volume with stress and strain tensors, cannot explain the experimentallyobserved drastic decrease in PT pressure caused by shear stresses or plastic strains. The main reason for this is that the shear stresses are limited by an engineering (macroscopic) yield strength, e.g. $1 \mathrm{GPa}$, and its contribution to the thermodynamic driving force for PT is small in comparison with the hydrostatic pressure, e.g. $10 \mathrm{GPa}$.

No publications that explain and characterize any of the above mechanochemical phenomena were available before 2004, and the actual physical mechanisms remain unknown. Even the fundamental dissimilarity between the pressureinduced PTs under quasi-hydrostatic loading and plastic strain-induced PTs at elevated pressure was not realized prior to the appearance of our papers. ${ }^{24,25)}$ Pressure- and stressinduced phase transformations start at crystal defects that naturally exist in a material and when stresses do not reach the yield limit. These defects (e.g. various dislocation structures or grain boundaries) produce stress concentrators and serve as nucleation sites for a phase transformation. First, nucleation occurs at defects with the strongest stress concentration; pressure should then be increased to cause nucleation at defects with decreasing level of generated stresses. An overview for analysis and characterization of the pressure-induced and stress-tensor-induced PTs can be found in Ref. 32),

Plastic strain-induced phase transformations take place by nucleation and limited growth at defects, produced in the course of plastic straining. The largest stress concentration can be produced at the tip of the dislocation pileups. Indeed, all stress components (and, consequently, pressure) $\sigma_{i j} \sim N$, where $N$ is the number of dislocations in a pileup. Since $N=10-100$, local stresses could be huge and can be increased by growing plastic deformation at relatively low pressure, thus driving phase transformations. An important point is that the deviatoric (nonhydrostatic) stresses near the defect tip are not bounded by the engineering yield strength but rather by the ideal strength in shear for a defect-free lattice, which may differ by a factor of 10 to 100 . Local stresses of such magnitude may result in nucleation of the high-pressure phase at an applied pressure that is not only significantly lower than that under hydrostatic loading but also below the phase-equilibrium pressure. In addition, such highly-deviatoric stress states with large stress magnitudes cannot be realized in bulk. Such unique stresses may lead to PTs into stable or metastable phases that were not or could not be attained in bulk under hydrostatic or quasi-hydrostatic conditions. It was concluded ${ }^{24,25)}$ that plastic strain-induced transformations require very different thermodynamic, kinetic, and experimental treatments than pressure- and stress-induced transformations.

\section{Crystal Lattice Instability Criteria: Phase Field Approach, Molecular Dynamics, and First Principle Approaches}

Barrierless nucleation during displacive PT usually occurs due to local lattice instability. Therefore, one must find the thermomechanical instability criteria for a perfect lattice under the general stress tensor, which will represent the PT criteria for perfect lattices. This seemed to be impossible due to the large number of combinations, but unexpected guidance came from the PT criterion analytically formulated within the large-strain phase field approach (PFA) ${ }^{33)}$ under action of all six stresses $\sigma_{i j}$. Molecular dynamics simulations ${ }^{34,35)}$ and the first-principle simulations ${ }^{36)}$ were then performed to find lattice instability conditions for cubic-totetragonal PT between diamond cubic phase Si I and metallic phase Si II, in both directions. The results for $\mathrm{Si} \mathrm{I} \rightarrow \mathrm{Si}$ II PT obtained with molecular dynamics and first-principle simulations are quite close. The lattice instability criteria for direct and reverse PTs are both found to be linear in normal-to-cubic-faces stresses $\sigma_{i}$, in accordance with the PFA predictions. Each criterion contains only two material parameters, which can be determined by atomistic simulations for two different normal stress states. Through geometrically-nonlinear terms that reflect change in the shape of the sample due to shear, these criteria are slightly dependent on the shear stresses at cubic faces. The strong effect of deviatoric stresses is found (Fig. 2). In particular, the mean stress required for PT from $\mathrm{Si}$ I to $\mathrm{Si}$ II under uniaxial compression is lower by a factor of 21 than that under hydrostatic loading. While the required non-hydrostatic stresses cannot be achieved in the actual defective bulk material because the macroscopic yield strength is much lower, they can be generated by the dislocation pileup with large $N$, which will lead to the experimentally-observed applied PT pressure.

In addition, atomistic simulation provided information, material functions and parameters for developing and calibrating our phase field models. ${ }^{37,38)}$ 


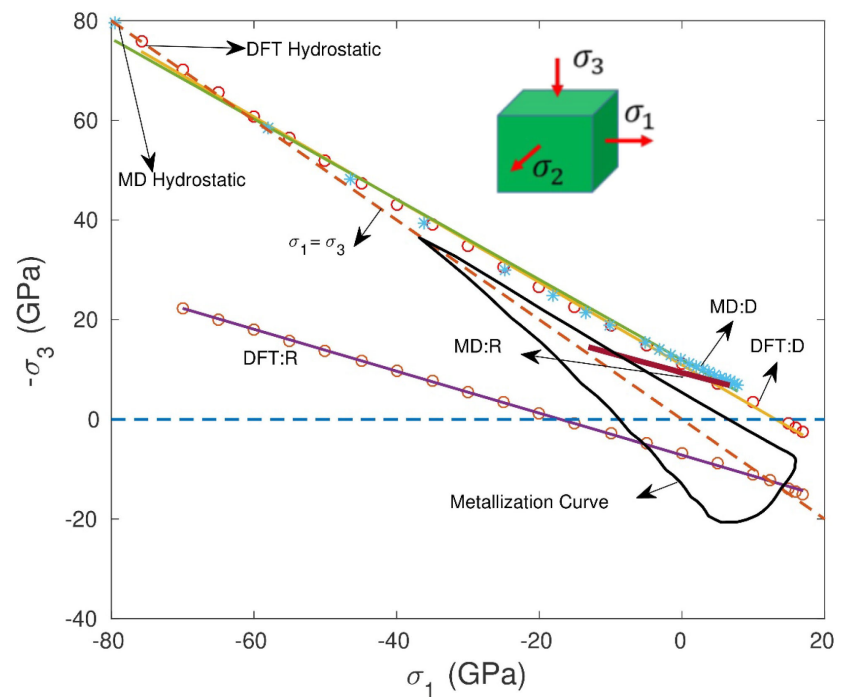

Fig. 2 Phase transformation criteria in terms of stress $\sigma_{3}$ vs. $\sigma_{1}=\sigma_{2}$ for direct (D) Si I-to-Si II and reverse (R) Si II-to-Si I phase transformations from the first-principle simulations and molecular dynamics simulations, ${ }^{34,35)}$ as well as the metallization criterion from the first-principle simulations. This figure is reproduced with permission from Ref. 36).

\section{Phase Nucleation at Dislocation Pileups}

An analytical solution for barrierless nucleation of the HPP at the tip of the dislocation pileup ${ }^{24,25)}$ confirmed our hypothesis that for large enough $N$, nucleation pressure may be lower, by a factor of 10 , than under hydrostatic loading with a single dislocation. However, the problem formulation was oversimplified, including linear elasticity, small strain formulation, infinite space, and no stress relaxation at the tip of the dislocation pileup. For a much more realistic treatment, the first PFA for the coupled dislocation and phase structure evolution was suggested, ${ }^{31,40-42)}$ this approach synergistically combines the most advanced large-strain theories for martensitic PTs ${ }^{33,43,44)}$ and dislocations, ${ }^{45,46)}$ with multiple interaction terms. The developed PFA was implemented in a finite element method (FEM) code COMSOL and was utilized for the first study of nucleation and growth of the HPP under normal stress and shear strain (Fig. 3) ) $^{31,39,41,42)}$ in a bicrystal. The obtained results prove that the superimposed plastic shear can reduce PT pressure by a factor of 10 and more.
However, plasticity does not only facilitate PT by generating stress concentrators (Fig. 3(a)); it also relaxes these stresses at the tip of the dislocation pileup, reducing the promoting effect (Fig. 3(b)). Some examples of finding loading processes that allow PT to overcome dislocation plasticity can be found in Ref. 31).

For a neglected jump in elastic moduli and surface energy, the thermodynamic condition of phase equilibrium for any material points of a phase interface is

$$
\sigma_{i j} \varepsilon_{i j}^{t}=\Delta G^{\theta} .
$$

Here, $\sigma_{i j} \varepsilon_{i j}^{t}$ is the transformation work, which is the sum of the product of the components of stress tensor $\sigma_{i j}$ and corresponding components $\varepsilon_{i j}^{t}$ of the transformation-strain tensor, and $\Delta G^{\theta}$ is the jump in the thermal part of the free energy. For the obtained stress tensor fields, the contour lines corresponding to the thermodynamically-equilibrium value of the transformation work are presented in Fig. 3(a) and (b). It is clear that the local phase-equilibrium condition is satisfied for the major parts of the phase interfaces. Note that a similar criterion is also approximately fulfilled for stresses averaged over the HPP or the grains. ${ }^{39,41,42)}$ These results are very important for the developing microscale coarse-grained theories.

Microscale PFA to multivariant martensitic PTs was developed, ${ }^{47,48)}$ combined with discrete dislocation evolution within shear bands utilizing contact problem formulation, ${ }^{49)}$ and used for the modeling of high-pressure phase evolution at dislocation pileups. Microscale PFA reproduces results of the nanoscale PFA shown in Fig. 3(a) and (b) reasonably well. In addition, due to its scale-free nature, it was also applied for a much larger sample.

\section{Retaining of Metastable High-Pressure Phases under Ambient Pressure}

This is the key goal if one wants to utilize the metastable HPPs for any real-world applications. The first step is to confirm that, for a given phase, the stress-free state does not meet the PT conditions under the stress tensor for the reverse PT to a low-pressure phase (LPP), obtained using atomic simulations; ${ }^{34-36)}$ then the phase is indeed a metastable one rather than unstable and can in principle be arrested at atmospheric pressure. The best way is to produce complete

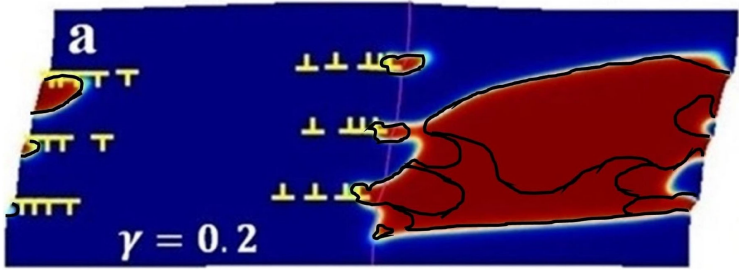

(a)

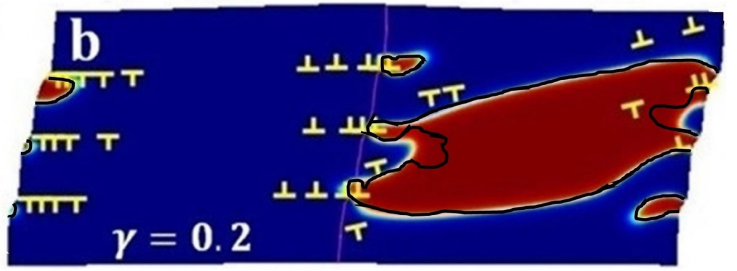

(b)

Fig. 3 Stationary solutions for interaction of the phase transformation to a HPP (red color) and dislocations in a bicrystalline sample subjected to normal stress and shear strain, without (a) and with (b) plasticity in the right grain. Dislocation pileup causes PT at pressure (averaged over the grain) significantly smaller than under hydrostatic loading with a single dislocation. Dislocations promote PT by producing stress-tensor concentrators (a) but also suppress PT through relaxation of stresses near the tip of the dislocation pileup (b). The contour lines of the equilibrium PT work are presented in black and, in most cases, coincide with the stationary phase interfaces. This figure is reproduced with permission from Ref. 39). 
PT to the HPP so that reverse PT would require nucleation, which may have a high barrier. If nucleation can occur or if residual LPP is unavoidable, growth of the LPP should be suppressed by producing obstacles to interface motion, increasing its athermal (threshold-type) friction. This can be done by plastically deforming the HPP above $p_{\varepsilon}^{r}$, pinning interfaces by dislocations, grain boundaries, point and other defects. These defects all produce resistance to the interface motion. ${ }^{50)}$ At the same time, these defects may promote nucleation of LPP in some cases by producing stress concentration or changing chemical energy. Reduction in the grain size also increases pressure hysteresis and allows one to retain metastable HPP, e.g. in oxides $\mathrm{ZrO}_{2}, \mathrm{Y}_{2} \mathrm{O}_{3}$, $\mathrm{BaTiO}_{3}, \mathrm{Al}_{2} \mathrm{O}_{3}, \mathrm{TiO}_{2}$, and iron oxides. ${ }^{29)}$ From the macroscopic point of view, the pressure hysteresis for pressureand stress-induced PT is proportional to the current value (under current plastic strain) of the yield strength. . $^{24,25,51 \text { ) }}$ Plastic strain and grain refinement increase (until saturation) the yield strength and, consequently, pressure hysteresis, which may prevent the reverse PT. ${ }^{24,25)}$ One must also find an unloading process of the sample in anvils, which minimizes or completely eliminates plastic straining below $p_{\varepsilon}^{r}$, minimizing or eliminating strain-induced reverse PT. ${ }^{52,53)}$

\section{Thermodynamically Consistent Kinetics of Plastic Strain Induced Phase Transformations at the Micro- scale}

A thermodynamically-consistent kinetic equation for the volume fractions of an HPP was derived in Refs. 24, 25) for the two-phase systems and in Ref. 54) for the multiphase systems. It was motivated by the results of our nanoscale studies, specifically by solutions of problems on nucleation at dislocation pileup ${ }^{24,25)}$ and nucleation at the shear-band intersection in TRIP steel ${ }^{55}$ ) at normal pressure. An additional thermodynamic driving force due to a strain-induced pressure concentrator was included and connected to plastic strain. Since dislocation pileups cause stresses of both signs of the same magnitude in regions with extra and missing atomic planes, respectively, they facilitate direct and reverse PTs in corresponding regions at the same time. This is included in the kinetic equation in terms of similar contributions for direct and reverse PTs. Because of the experimental results summarized in item 4 of Section 1, time is not an important parameter and is substituted with the accumulated plastic strain $q$. Also, it is taken into account that plastic strain is larger (or smaller) in the phase with the smaller (or larger) yield strength.

Among multiple material parameters in the kinetic equation, two are the most important: $p_{\varepsilon}^{d}$, the minimum pressure below which a plastic strain-induced PT from LPP to HPP is impossible, and $p_{\varepsilon}^{r}$, the maximum pressure above which a strain-induced PT from HPP to LPP is impossible.

For PTs that are strongly affected by plastic strain, i.e. for which $p_{\varepsilon}^{d}<p_{\varepsilon}^{r}$, and for pressure $p_{\varepsilon}^{d}<p<p_{\varepsilon}^{r}$, a stationary solution for the kinetic equation is shown in Fig. 4. The prediction in Refs. 24, 25, 54) of the existence of a stationary two-phase solution (and, more generally, stationary $n$-phase solution in Ref. 54)) and its independence of the loading history is confirmed experimentally in Ref. 56) by observed

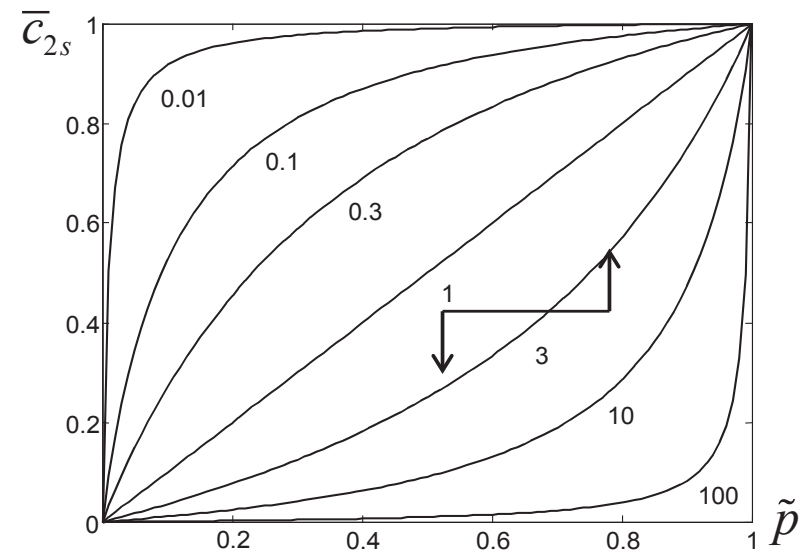

Fig. 4 The stationary value of the volume fraction of the HPP vs. dimensionless pressure $\tilde{p}=\frac{p-p_{\varepsilon}^{d}}{p_{\varepsilon}^{r}-p_{\varepsilon}^{d}}$. Numbers near curves designate values of a parameter $M$, which is proportional to the ratio of the yield strengths in compression of the HPP and LPP. For any pressure $\tilde{p}$ and volume fraction of the HPP corresponding to a stationary value $\bar{c}_{2 s}$, any infinitesimal pressure decrease (or growth) followed by plastic shearing will result in PT to LPP (or HPP) until a new stationary state is reached. This means an infinitesimal difference between pressures for direct and reverse PTs, i.e. pressure hysteresis. This result is in agreement with the experiments in Refs. 7, 30). Reproduced with permission from Ref. 25).

incompletion of various PTs, and by the existence of the stationary state independent of the processing history (equifinality). In turn, existence of the stationary solution with incomplete PT means that $p_{\varepsilon}^{d}<p_{\varepsilon}^{r}$ for a given PT and that PT is strongly affected by plastic strain. Note that another contribution to the incompleteness of a PT may come from heterogeneous stress-strain fields in a sample in rotational diamond anvils.

As follows from Fig. 4, the stationary volume fraction of the HPP grows with the increasing ratio of the yield strengths in compression of the HPP and LPPs. This means that superimposing plastic shear under pressure is a promising way to promote strong and superhard HPPs. Intuitively, this is not surprising, because plastic deformation is larger in the weaker phase and smaller in the stronger phase. Weak phases cannot be obtained in large amounts for pressure slightly higher than $p_{\varepsilon}^{d}$; this can only be done close to the pressure $p_{\varepsilon}^{r}$ (Fig. 4). Note that the above discussion is only valid for $p_{\varepsilon}^{d}<p_{\varepsilon}^{r}$.

Figure 4 allows us to interpret the absence of the pressure hysteresis found experimentally for $\alpha \leftrightarrow \omega \mathrm{PT}$ in zirconium and titanium ${ }^{57)}$ in rotational Bridgman anvils as well as for $\mathrm{B}_{1}$ to $\mathrm{B}_{2} \mathrm{PT}$ in potassium chloride ${ }^{1)}$ in rotational diamond anvils. This does not, however, mean that the phase-equilibrium pressure is determined, because a zero-pressure hysteresis can be obtained for any pressure in the range $p_{\varepsilon}^{d}<p<p_{\varepsilon}^{r}$.

In the opposite case of $p_{\varepsilon}^{d}>p_{\varepsilon}^{r}$, a stationary solution, which can be reached for large plastic strains, is very simple: the complete LPP exists below $p_{\varepsilon}^{d}$, the complete HPP can only be found above $p_{\varepsilon}^{r}$, and strain-induced PTs do not occur in the range $p_{\varepsilon}^{r}<p<p_{\varepsilon}^{d}$. Thus, two-phase stationary mixture and zero-pressure hysteresis are impossible for this case.

Kinetics of multiphase strain-induced PTs was analyzed in Ref. 54). One interesting result for PTs in silicon was related to a non-monotonous variation of the volume fraction of 
metallic Si II during plastic straining at fixed pressure. While Si II is practically absent in the stationary solution, which is reached for very large plastic strain, it is detectable at relatively small plastic strains. The same may happened for some hidden (unknown) phases to be searched, meaning that the generic wisdom that the plastic strain should be maximal is not true. Plastic strain should be optimal. There are various other reasons for this statement, one of which is found ${ }^{21)}$ for $\mathrm{PT}$ from hexagonal $\mathrm{BN}$ to superhard wurtzitic $\mathrm{BN}$; this is related to an increase in the concentration of the turbostratic stacking fault with the plastic strain, which strongly suppresses PT. Another reason is related to the macroscopic behavior of the sample, specifically to reduction in the width of a sample in the course of rotation of an anvil, ${ }^{25)}$ which reduces the mass of the HPP.

\section{Macroscale Modeling of Combined Plastic Flow and Phase Transformation in a Sample in Rotational Anvils}

\subsection{Modeling of plastic flow}

It was found ${ }^{58)}$ for more than 60 materials (simple metals and alloys, minerals, oxides, etc.) that, for monotonous and quasi-monotonous loadings and plastic strain exceeding a certain critical value, the initially-isotropic polycrystals deform as the perfectly plastic (i.e. without strain hardening or softening) and isotropic material with a strain-historyindependent limiting surface of the perfect plasticity. Some additional confirmations are presented for alloyed steel and $\mathrm{NaCl}^{4}{ }^{4}$ Saturation of the strain hardening is now generally accepted in the severe plastic deformation community. ${ }^{8,59)}$

However, the results in Ref. 58) are much broader because they include independence of the strain history and isotropic behavior, which is exactly opposite to what was expected from elastoplastic behavior. It is shown in Ref. 58) how to develop an elastoplasticity theory that includes strain and strain history dependence and plastic strain-induced anisotropy, which disappear for monotonous and quasimonotonous loadings. This experimental regularity significantly simplifies the theory and experimental calibration of the specific models.

Note that the value of the threshold plastic strain for disappearance of strain hardening, $m$, determined from highpressure-torsion experiments (which is in the range of $m=5$ to 20 for copper), is highly overestimated ${ }^{60)}$ because the relationship between torque and anvil rotation angle is weakly dependent on the value of $m$ (Fig. 5). However, relationship between torque and rotation angle is strongly dependent on the Coulomb friction coefficient between the flash and anvil. This was neglected in the interpretation of experimental data because of the relatively small width of the flash. For this reason, stress-strain curves extracted from torque-rotation angle curves contain significant errors. Values of $m$ obtained in uniform compression for six metals ${ }^{61)}$ range from 0.44 for cast iron to 1.35 for low-carbon steel to 1.57 for copper.

Analytical solutions for high-pressure torsion in unconstrained ${ }^{24,25)}$ and constrained ${ }^{62)}$ configurations are found to be in good correspondence with FEM solutions in Refs. 63 and $62)$, respectively. There are numerous other FEM solutions

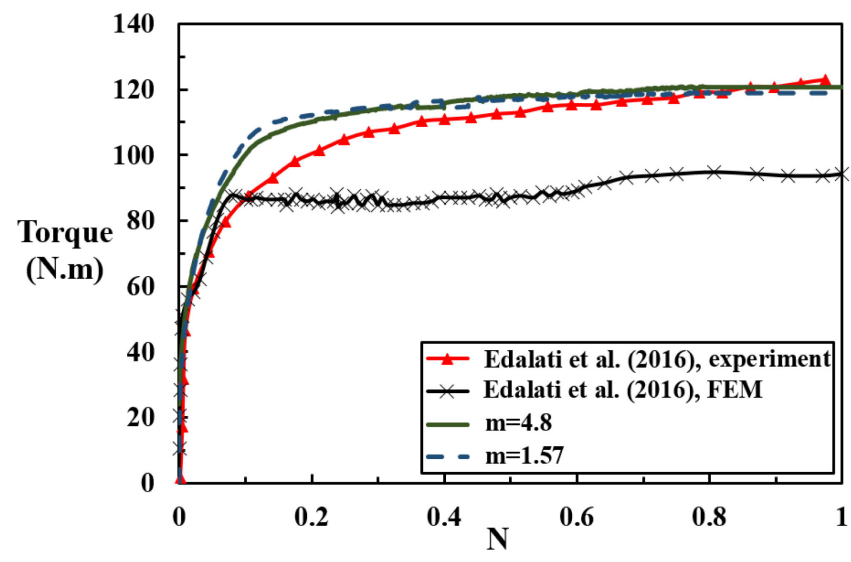

Fig. 5 Relationship between the torque and number of revolutions of the anvil for two critical strains for saturation of strain hardening, $m=4.8$ and $m=1.57$, obtained in Ref. 60), and the same relationship from the experimental and FEM results in Ref. 9). It is clear that the results are weakly dependent on the magnitude of $m$. Reproduced with permission from Ref. 60).

for high-pressure torsion at relatively low pressure, e.g..$^{964-66)}$ These are based either on a Coulomb friction model or full adhesion, which introduces some error in a solution. A combined Coulomb friction and plastic sliding model, for which the magnitude of the friction stress is equal to the yield strength in shear, is utilized for high-pressure torsion of copper $^{60)}$ and for plastic flow with PTs. ${ }^{67,68)}$

One argument in justifying the complete adhesion condition in the recent works on high-pressure torsion is due to surface roughness. However, even for complete adhesion, localized shear flow in the thin layer of the deformed materials just below the anvil's asperities is equivalent to the plastic sliding. Note that, even under complete adhesion conditions, analytical ${ }^{24,25)}$ and numerical $^{63)}$ solutions both exhibit plastic sliding. However, a very fine mesh is needed to capture it. Therefore, introducing plastic sliding conditions through the contact problem ${ }^{60}$ minimizes this numerical error and allows one, in particular, to better reproduce the experimental torque-rotation angle curve.

In Ref. 69), the problem on compression of a rhenium sample in diamond anvils was solved using FEM and based on the developed large elastic and plastic strain elastoplastic pressure-dependent model with nonlinear elasticity. The obtained pressure distribution is in good correspondence with experiments for pressures up to $300 \mathrm{GPa}^{22)}$ The same elastoplastic model was used, with a more general contact sliding rule, for torsion in rotational diamond anvils. ${ }^{70,71)} \mathrm{A}$ pressure-self-focusing effect, consisting of a very large pressure gradient and, consequently, high pressure in the center of a diamond anvil with a beveled culet, was revealed. Analysis led to suggestions on how to utilize the pressure self-focusing to reach the maximum possible pressure in traditional and rotational diamond anvils.

\subsection{Modeling and simulation of plastic deformation and} strain-induced phase transformations

A macroscale phenomenological model for combined plastic flow and strain-induced PTs, including microscale 


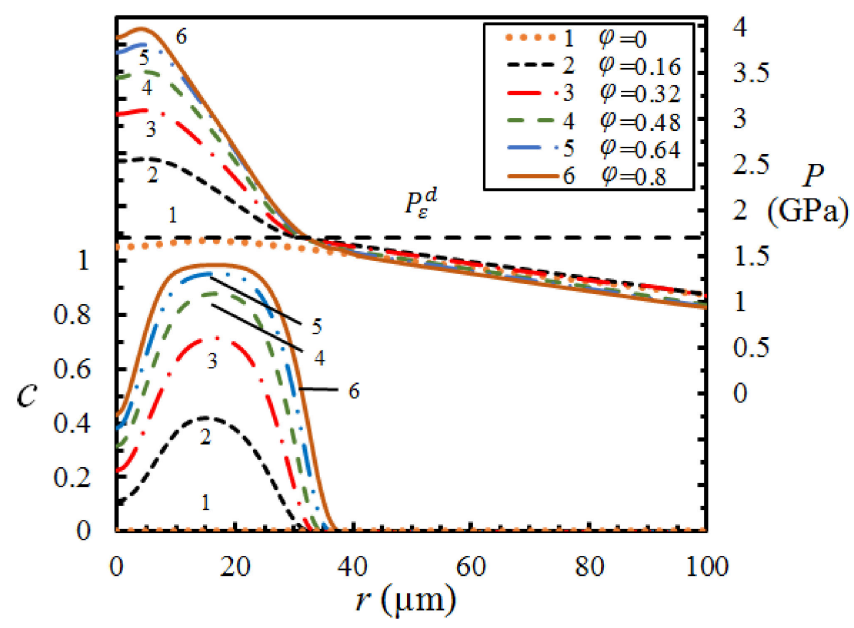

Fig. 6 Distributions of the pressure $p$ and concentration $c$ of the $\omega$ phase at the contact surface between a zirconium sample and diamond anvil, in the region $0 \leq r \leq 100 \mu \mathrm{m}$, in the course of twisting under the fixed axial force. Rotation angle of an anvil $\varphi$ is expressed in radians. Reproduced with permission from Ref. 72).

strain-controlled kinetics (described in Section 6), was developed and applied for simulation of a sample compressed and then twisted under constant load in rotational diamond anvils. In the first series of papers, ${ }^{52,53,67,68,72-77)}$ the effect of pressure on mechanical properties, finite elastic and transformational deformations, as well as elastic nonlinearity were neglected. Strain hardening was also neglected for reasons summarized in Section 7.1. Full adhesion at the contact surface between diamond and a sample was assumed in Refs. 52, 53, 73-77); contact sliding utilizing combined Coulomb and plastic friction was introduced in Refs. 17, 67, $68,72)$. Here, we will present some recent simulations for plastic strain-induced $\alpha \rightarrow \omega$ transformation in zirconium under torsion in rotational diamond anvils. Fields of the pressure $p$, concentration $c$ of the $\omega$ phase, and plastic strain $q$ at the contact surface and in the bulk of the sample in the course of twisting under the fixed applied force are presented in Figs. 6 and 7. Note that the yield strength of the $\omega$ phase is greater than that of the $\alpha$ phase by a factor of 5.56, according to experiments.

During rotation of an anvil, PT occurs in the approximately cylindrical region where $p>p_{\varepsilon}^{d}$, which practically does not grow. The growth of the pressure gradient in the transforming region is caused by the increase in the yield strength of mixture during PT progress, despite the constant applied force and compressive volumetric transformation strain. Such a pressure growth was first obtained experimentally for PT in potassium chloride ${ }^{7,19)}$ and fullerene ${ }^{19,20)}$ and named the "pressure self-multiplication effect". While this effect formally contradicts the principle of Le Shatelie, it in fact does not because, as was previously mentioned, plastic straininduced PTs are described by more complicated thermodynamics and kinetics. They are driven by plastic strain rather than pressure, which should just exceed $p_{\varepsilon}^{d}$. Initially, within an oversimplified elastic model, it was explained by an increase in the elastic moduli during the PT. ${ }^{7}$ However, more adequate analytical plastic solutions ${ }^{24,25,72)}$ suggested that the higher yield strength of the HPP and reduction in sample thickness during torsion, compensating for transformational
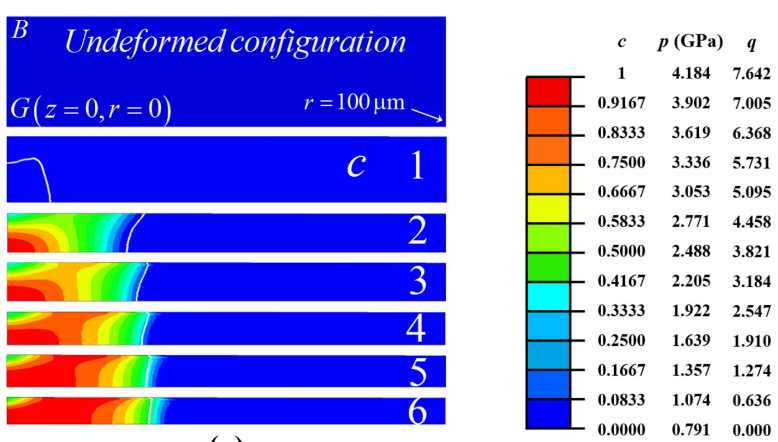

(a)

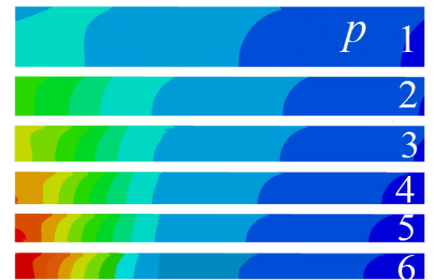

(b)

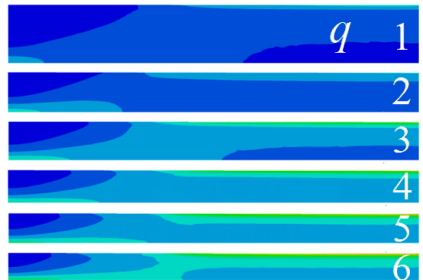

(c)
Fig. 7 Fields of the concentration $c$ of the $\omega$ phase (a), mean pressure $p(b)$, and plastic strain $q$ (c) in the bulk of the sample under the same conditions as in Fig. 6. The white line in Figure (a) corresponds to the minimum pressure for the PT to HPP $p_{\varepsilon}^{d}$. Reproduced with permission from Ref. 72).

volume decrease, are responsible for this effect. This was later confirmed by a much more detailed FEM solution. ${ }^{73}$ )

By increasing pressure during torsion, this effect increases the driving force and promotes the PT. However, it is not very practically useful for promotion of the PT due to pressure growth in the region when it was high enough for completion of PT at larger twisting. High pressure in the region where it is not required increases the probability of fracture of anvils. Such unwanted growth of pressure and pressure heterogeneity during PT can be reduced by using a gasket with optimized geometric parameters and properties; see experiments in Ref. 21) and simulation results in Refs. 68, 78).

It is clear from Fig. 7 that further rotation would not essentially increase the amount of the $\omega$ phase. Indeed, the region with $p>p_{\varepsilon}^{d}$ is almost fully occupied with the HPP and the sample thickness decreases during torsion, pushing the the $\omega$ phase to the region with $p<p_{\varepsilon}^{d}$, where, in principle, the $\omega \rightarrow \alpha$ PT may take place. The only way to increase the amount of the $\omega$ phase is to apply a larger force, increasing the region with $p>p_{\varepsilon}^{d}$. Note that finding material in the region with $p<p_{\varepsilon}^{d}$ (Fig. 7(a)) in an experiment may lead to error in the determination of $p_{\varepsilon}^{d}$ if one assumes that $p_{\varepsilon}^{d}$ is the lowest pressure where the $\omega$ phase is detected.

Strong pressure heterogeneity in Figs. 6 and 7 leads to the conclusion that transformation pressure cannot be characterized based on the averaged pressure (force over total area). This was also mentioned for compression in Ref. 77). However, all high-pressure torsion experiments ${ }^{10,11,72)}$ use the averaged pressure for this purpose. Based on FEM results, such an averaged pressure should be corrected by a factor of 2.2 for strain-induced $\alpha \rightarrow \omega$ transformation and by a factor of 5 for strain-induced $\omega \rightarrow \beta$ transformation to obtain a correct estimate for $p_{\varepsilon}^{d}$; see Ref. 72).

All previous limitations of the model in Refs. 52, 53, 67, $68,72-77)$ were eliminated in Refs. 78, 79). A general model 
for coupled PT and plasticity with large plastic, transformational, and elastic deformations was developed. It includes the same strain-controlled microscale kinetic equation, the Murnaghan elastic rule, and pressure-dependent yield strength. All elastic and plastic material constants are linear functions of the volume fraction of HPP. A finite-strain, anisotropic, and nonlinear single-crystal elastic rule was utilized for diamond anvils. Strain-induced PT from graphitelike to wurtzitic BN within a rhenium gasket was studied under compression in traditional diamond anvils up to $50 \mathrm{GPa}^{78)}$ and under torsion in rotational diamond anvils. ${ }^{79)}$ Cases of probable misinterpretation of the experimental measurements and characterization of the strain-induced PTs are analyzed. The obtained results offer a valuable insight into combined PTs and plastic flow in various materials in rotational diamond anvils, and are crucial for the planning of an optimum experimental program and the calibration of material parameters for PT, as well as for the control of PTs by changing the geometric and mechanical parameters of a gasket.

\section{Summarizing Remarks}

Four major experimental effects observed under torsion of materials at fixed force in rotational Bridgman or diamond anvils are the possibilities (a) to lower PT pressure by a factor of 2 to 10 and more in comparison to compression under quasi-hydrostatic conditions; (b) to obtain hidden (new) metastable phases and compounds that have not been attained under quasi-hydrostatic compression; (c) to retain metastable high-pressure phases under normal pressure; and (d) to reduce the pressure hysteresis, even to zero. Traditional thermodynamics, even after substituting pressure and volume with stress and strain tensors, cannot explain these effects. This is related to the fact that the deviatoric stresses are limited by the macroscopic yield strength and their contribution to the driving force for the PT is much smaller than the contribution from the pressure. Our goal is to collect the results of our theory and simulations, at four scales from atomistic to macroscale, and present our current understanding of the underlying phenomena. Atomistic simulations using both classical and quantum mechanics provide critical conditions for the initiation of PTs in a perfect lattice for the general stress tensor. In particular, PT from Si I to Si II under uniaxial compression starts at a pressure 21 times lower than that under hydrostatic conditions.

A nanoscale mechanism of HPP nucleation at the tip of plastic strain-induced dislocation pileups is suggested as the most probable and is studied analytically and with developed PFA for interaction between discrete dislocations and PT. The magnitudes of all stress tensor components, including mean pressure and stress deviator, are proportional to the number of dislocations in a dislocation pileup and can be very large. Note that the deviatoric stresses near the defect tip are not bounded by the engineering yield strength but rather by the ideal strength in shear for defect-free lattice, which may differ by a factor of 10 to 100 . Such huge local stresses, which satisfy the PT criterion for a perfect lattice, may cause nucleation of the HPP at an applied pressure not only significantly below than that under hydrostatic loading but also lower than the phase-equilibrium pressure. The transformation pressure can indeed be lowered by an order of magnitude and more. In addition, such highly-deviatoric stress states with large stress magnitudes cannot be realized in bulk. Such unique stresses may lead to PTs into stable or metastable phases that were not or could not be attained in bulk under hydrostatic or quasi-hydrostatic conditions. However, plasticity may also relax stresses at the tip of defects, diminishing their nucleation potencies. Certain combinations of pressure and shear stress for which the PT is the dominant relaxation mechanism were established.

After careful experimental confirmation, the newlyobtained understanding of the nanoscale mechanisms for plastic strain-induced PTs may result in novel synthetic paths and technologies for material synthesis of new materials at the lowest possible pressure level. This understanding should be supplemented by microscale and macroscale theoretical, computational, and experimental approaches. They will allow: (a) physically-based characterization of plastic straininduced PTs in terms of corresponding kinetic equations; (b) collection of information about heterogeneity of all relevant fields at these scales; (c) and optimal design of geometric and mechanical parameters of a gasket to achieve the desired local loading and transformation paths. After this, processes in rotational diamond anvils can be scaled up using highpressure torsion with ceramic or metallic anvils.

For the material with a very strong effect of plastic strain on PT (i.e. for which $p_{\varepsilon}^{d}<p_{\varepsilon}^{r}$ ), our microscale kinetic equation: (a) predicted a stationary two- or multiphase solution and its independence of the loading history, which was confirmed experimentally; and (b) explained the zeropressure hysteresis observed experimentally. Lack of hysteresis, however, does not mean that the phase-equilibrium pressure is determined, because it can be obtained for any pressure in the range $p_{\varepsilon}^{d}<p<p_{\varepsilon}^{r}$.

One goal of the synthesis of the metastable HPP is to retain them at room pressure and utilize them for practical purposes. Multiscale theory suggests the following ways to do this: (a) to produce a complete PT to the HPP phase so that reverse PT would require nucleation; (b) to plastically deform the HPP above $p_{\varepsilon}^{r}$, pinning interfaces by dislocations, point defects, grain boundaries, and other defects; (c) and to find an unloading process of the sample in the anvils which minimizes/eliminates plastic straining below $p_{\varepsilon}^{r}$, thus minimizing/eliminating strain-induced reverse PT.

\section{Acknowledgements}

The author acknowledges support of US Army Research Office (W911NF-12-1-0340, manager Dr. David Stepp), National Science Foundation (CMMI-1536925, manager Dr. Siddiq Qidway), Office of Naval Research (N00014-191-2082, manager Dr. Chad Stolz), and Iowa State University (Vance Coffman Faculty Chair Professorship).

\section{REFERENCES}

1) P.W. Bridgman: Phys. Rev. 48 (1935) 825-847.

2) P.W. Bridgman: Studies in Large Plastic Flow and Fracture, (McGrawHill, New York, 1952).

3) P.W. Bridgman: Proc. Am. Acad. Arts Sci. 71 (1937) 387-460. 
4) R.Z. Valiev, R.K. Islamgaliev and I.V. Alexandrov: Prog. Mater. Sci. 45 (2000) 103-189.

5) A.P. Zhilyaev and T.G. Langdon: Prog. Mater. Sci. 53 (2008) 893-979.

6) R.Z. Valiev, Y. Estrin, Z. Horita, T.G. Langdon, M.J. Zehetbauer and Y.T. Zhu: JOM 58(4) (2006) 33-39.

7) V.D. Blank and E.I. Estrin: Phase Transitions in Solids under High Pressure, (CRC Press, New York, 2014).

8) K. Edalati and Z. Horita: Mater. Sci. Eng. A 652 (2016) 325-352.

9) K. Edalati, D.J. Lee, T. Nagaoka, M. Arita, H.S. Kim, Z. Horita and R. Pippan: Mater. Trans. 57 (2016) 533-538.

10) B. Srinivasarao, A.P. Zhilyaev and M.T. Perez-Prado: Scr. Mater. 65 (2011) 241-244.

11) A.P. Zhilyaev, I. Sabirov, G. Gonzaalez-Doncel, J. Molina-Aldareguia, B. Srinivasarao and M.T. Perez-Prado: Mater. Sci. Eng. A 528 (2011) 3496-3505.

12) K. Edalati, M. Arimura, Y. Ikoma, T. Daio, M. Miyata, D.J. Smith and Z. Horita: Mater. Res. Lett. 3 (2015) 216-221.

13) K. Edalati, S. Toh, Y. Ikoma and Z. Horita: Scr. Mater. 65 (2011) 974 977.

14) Y. Ivanisenko, A. Kilmametov, H. Rosner and R.Z. Valiev: Int. J. Mater. Res. 99 (2008) 36-41.

15) A. Zharov: Usp. Khim. 53 (1984) 236-250.

16) A. Zharov: Reaction of Solid Monomers and Polymers under Shear Deformation and High Pressure. High Pressure Chemistry and Physics of Polymers chapter 7, ed. by Kovarskii, (CRC Press, Boca Raton, 1994) pp. 267-301.

17) V.D. Blank, Y.S. Konyaev, A.I. Kuznetsov and E.I. Estrin: Instrum. Exp. Tech. 27 (1984) 1240-1242.

18) N.B. Novikov, L.K. Shvedov, Y.N. Krivosheya and V.I. Levitas J. Superhard Mater. 37 (2015) 1-7.

19) N.V. Novikov, S.B. Polotnyak, L.K. Shvedov and V.I. Levitas: J. Superhard Mater. 21 (1999) 36-48.

20) V.D. Blank, M. Popov, S. Buga, V. Davydov, V.N. Denisov, A.N. Ivlev, B.N. Marvin, V. Agafonov, R. Ceolin, H. Szwarc and A. Rassat: Phys. Lett. A 188 (1994) 281-286.

21) V.I. Levitas, Y. Ma, J. Hashemi, M. Holtz and N. Guven: J. Chem. Phys. 125 (2006) 044507.

22) R.J. Hemley, H.K. Mao, G.Y. Shen, J. Badro, P. Gillet, M. Hanfland and D. Hausermann: Science 276 (1997) 1242-1245.

23) V.I. Levitas, Y. Ma, E. Selvi, J. Wu and J. Patten: Phys. Rev. B 85 (2012) 054114

24) V.I. Levitas: Continuum Mechanical Fundamentals of Mechanochemistry. High Pressure Surface Science and Engineering section 3, ed. by Y. Gogotsi and V. Domnich, (Inst. of Physics, Bristol, 2004) pp. 159 292.

25) V.I. Levitas: Phys. Rev. B 70 (2004) 184118

26) C. Ji, V.I. Levitas, H. Zhu, J. Chaudhuri, A. Marathe and Y. Ma: Proc. Natl. Acad. Sci. USA 109 (2012) 19108-19112.

27) V.I. Levitas and L.K. Shvedov: Phys. Rev. B 65 (2002) 104109.

28) V.L. Solozhenko: High Press. Res. 13 (1995) 199-214

29) K. Edalati: Adv. Eng. Mater. 21 (2019) 1800272.

30) V.I. Levitas: Proceedings of the International Conference on Martensitic Transformations: Chicago (ICOMAT 2017), plenary lecture, Chicago, IL, July 7-14, 2017. Ed. by A. Stebner, G. Olson, V. Levitas, et al., (The Minerals, Metals \& Materials Society, 2018) pp. $3-10$.

31) V.I. Levitas and M. Javanbakht: Nanoscale 6 (2014) 162-166.

32) V.I. Levitas: J. Phys. Condens. Matter 30 (2018) 163001.

33) V.I. Levitas: Int. J. Plasticity 49 (2013) 85-118.

34) V.I. Levitas, H. Chen and L. Xiong: Phys. Rev. Lett. 118 (2017) 025701 .

35) V.I. Levitas, H. Chen and L. Xiong: Phys. Rev. B 96 (2017) 054118.

36) N.A. Zarkevich, H. Chen, V.I. Levitas and D.D. Johnson: Phys. Rev. Lett. 121 (2018) 165701.

37) V.I. Levitas: Int. J. Plasticity 106 (2018) 164-185.

38) H. Babaei and V.I. Levitas: Int. J. Plasticity 107 (2018) 223-245.

39) M. Javanbakht and V.I. Levitas: J. Mater. Sci. 53 (2018) 13343-13363.
40) V.I. Levitas and M. Javanbakht: J. Mech. Phys. Solids 82 (2015) 287319.

41) M. Javanbakht and V.I. Levitas: J. Mech. Phys. Solids 82 (2015) 164185.

42) M. Javanbakht and V.I. Levitas: Phys. Rev. B 94 (2016) 214104

$43)$ V.I. Levitas, V.A. Levin, K.M. Zingerman and E.I. Freiman: Phys. Rev. Lett. 103 (2009) 025702.

44) V.I. Levitas: J. Mech. Phys. Solids 70 (2014) 154-189.

45) V.I. Levitas and M. Javanbakht: J. Mech. Phys. Solids 82 (2015) 345366.

46) M. Javanbakht and V.I. Levitas: Int. J. Solids Struct. 82 (2016) 95-110.

47) V.I. Levitas, A.V. Idesman and D.L. Preston: Phys. Rev. Lett. 93 (2004) 105701 .

48) S.E. Esfahani, I. Ghamarian, V.I. Levitas and P.C. Collins: Int. J. Solids Struct. 146 (2018) 80

49) V.I. Levitas, S.E. Esfahani and I. Ghamarian: Phys. Rev. Lett. 121 (2018) 205701.

50) G.B. Olson and M. Cohen: Dislocation Theory of Martensitic Transformations. In: Dislocations in Solids, ed. by F.R.N. Nabarro, 7, (Elsevier Science Publishers B V, 1986) pp. 297-407.

51) V.I. Levitas: J. Mech. Phys. Solids 45 (1997) 1203-1222.

52) B. Feng, O.M. Zarechnyy and V.I. Levitas: J. Appl. Phys. 113 (2013) 173514.

53) B. Feng, V.I. Levitas and O.M. Zarechnyy: Comput. Mater. Sci. 84 (2014) 404-416

54) V.I. Levitas and O.M. Zarechnyy: J. Phys. Chem. B 110 (2006) 1603516046

55) V.I. Levitas, A.V. Idesman and G.B. Olson: Acta Mater. 47 (1998) 219233.

56) B.B. Straumal, A.R. Kilmametov, Y. Ivanisenko, A.A. Mazilkin, O.A. Kogtenkov, L. Kurmanaeva, A. Korneva, P. Zieba and B. Baretzky: Int. J. Mater. Res. 106 (2015) 657-664.

57) V.A. Zilbershtein, N.P. Chistotina, A.A. Zharov, N.S. Grishina and E.I. Estrin: Fiz. Met. i Metalloved. 39 (1975) 445-447.

58) V.I. Levitas: Large Deformation of Materials with Complex Rheological Properties at Normal and High Pressure, (Nova Science Publishers, New York, 1996).

59) K. Edalati and Z. Horita: Mater. Trans. 51 (2010) 1051-1054.

60) M. Kamrani, V.I. Levitas and B. Feng: Mater. Sci. Eng. A 705 (2017) 219-230.

61) V.I. Levitas, I.E. Stashkevich and A.B. Nemirovskii: Strength Mater. 26 (1994) 676-680.

62) Y. Beygelzimer, R. Kulagin, L.S. Toth and Y. Ivanisenko: Beilstein J. Nanotechnol. 7 (2016) 1267-1277.

$63)$ V.I. Levitas and O.M. Zarechnyy: High Press. Res. 30 (2010) 653-669.

64) P.H.R. Pereira, R.B. Figueiredo, P.R. Cetlin and T.G. Langdon: IOP Conf. Series: Mater. Sci. Eng. 63 (2014) 012041.

65) Y. Song, W. Wang, D. Gao, E.Y. Yoon, D.J. Lee, C. Lee and H.S. Kim: J. Mater. Sci. 48 (2013) 4698-4704.

66) D.J. Lee, E.Y. Yoon, D.H. Ahn, B.H. Park, H.W. Park, L.J. Park, Y. Estrin and H.S. Kim: Acta Mater. 76 (2014) 281-293.

67) B. Feng, V.I. Levitas and O.M. Zarechnyy: J. Appl. Phys. 114 (2013) 043506.

68) B. Feng and V.I. Levitas: J. Appl. Phys. 119 (2016) 015902.

69) B. Feng, V.I. Levitas and R.J. Hemley: Int. J. Plasticity 84 (2016) 3357.

70) B. Feng and V.I. Levitas: Sci. Rep. 7 (2017) 45461.

71) B. Feng and V.I. Levitas: Int. J. Plasticity 92 (2017) 79-95.

72) B. Feng, V.I. Levitas and M. Kamrani: Mater. Sci. Eng. A 731 (2018) $623-633$.

73) V.I. Levitas and O.M. Zarechnyy: Phys. Rev. B 82 (2010) 174124.

74) V.I. Levitas and O.M. Zarechnyy: Phys. Rev. B 82 (2010) 174123.

$75)$ B. Feng and V.I. Levitas: J. Appl. Phys. 114 (2013) 213514.

76) B. Feng, V.I. Levitas and Y. Ma: J. Appl. Phys. 115 (2014) 163509.

77) B. Feng and V.I. Levitas: Mater. Sci. Eng. A 680 (2017) 130-140.

$78)$ B. Feng and V.I. Levitas: Int. J. Plasticity 96 (2017) 156-181.

79) B. Feng, V.I. Levitas and W. Li: Int. J. Plasticity 113 (2019) 236-254. 